Invited Editorial

4

6

7 The George Institute for Global Health, University of Oxford, Oxford, UK

8

9

10

11

12

13 Oxford Martin School

14 University of Oxford

1534 Broad Street

16 Oxford OX1 3BD

17 kazem.rahimi@georgeinstitute.ox.ac.uk

18

19

20

21

\author{
Kazem Rahimi
}

\section{failure research}


In 1972, Rubler et al. reported the clinical and post-mortem findings of a small cohort study involving 27 patients with diabetes. ${ }^{1}$ The vast majority of these patients (85\%) had several other cardiovascular risk factors, or evidence of ischaemic heart disease or valvular disease. After excluding these patients, the investigators found evidence of cardiomyopathy and heart failure in the remaining four patients with no apparent alternative cause other than diabetes. Intrigued by this somewhat puzzling finding, the authors coined the term "diabetic cardiomyopathy", implying that diabetes causes heart failure independent of other established cardiovascular risk factors.

Since that early report, several much larger studies have investigated the association between diabetes and risk of heart failure and other cardiovascular conditions. For instance, in a recent study of 35,000 UK adults with prevalent Type 2 diabetes without any known pre-

However, diabetes is not a single homogeneous disease. Among people with diabetes the risk of heart failure (and other vascular outcomes) differs substantially. In this issue of Heart, Dr Skrtic et al..$^{3}$ investigate the association between glycaemic control and risk of incident heart failure in one of the largest contemporary cohorts of patients with Type 2 diabetes. By making use of longitudinal Electronic Health Records of about 100,000 UK patients with Type 2 DM, they investigate the association between $\mathrm{HbA1c}$ as a marker of glycaemic control and risk of heart failure. During follow-up, about 6000 patients (6.4\%) were found to have incident heart failure. In their main analysis, a single measure of $\mathrm{HbA1c}$ that was Rahimi - DM and HF V1_2016-09-05 
recorded within 90 days prior and 30 days after the diagnosis of diabetes showed a continuous association with the risk of incident heart failure; more specifically, the risk of heart failure increased continuously by $6 \%$ for each 1 percentage point higher baseline HbA1c, after adjustment for several known confounders.

In additional analyses, the authors investigated the extent to which the observed associations varied by alternative measures of glycaemic control. ${ }^{3}$ In particular, they compared the predictive ability of a single $\mathrm{HbA1c}$ measurement at the time of diagnosis with the mean of all available $\mathrm{HbA} 1 \mathrm{c}$ measures (called updated mean $\mathrm{HbA1c}$ ). In a third analysis, they selected the latest $\mathrm{HbA} 1 \mathrm{c}$ value (called latest $\mathrm{HbA} 1 \mathrm{c}$ ) as the exposure variable. When the updated mean HbA1c measure was used, the pattern of association was very similar to the single baseline value with no evidence of a nadir. However, the associations were much stronger; the the risk of HF increased continuously by $15 \%$ (compared with $6 \%$ for baseline $\mathrm{HbA1c}$ ) for each 1 percentage point higher updated mean $\mathrm{HbA1c}$ difference. By contrast, when the latest $\mathrm{HbA} 1 \mathrm{c}$ value was chosen, there was an apparent $\mathrm{J}$-shaped association between $\mathrm{HbA} 1 \mathrm{c}$ and risk of heart failure with a nadir of $7-8 \% \mathrm{HbA} 1 \mathrm{c}$, below which the association seemed reversed.

How should we interpret these findings? First and perhaps most important, this study provides compelling evidence that poor glycaemic control is still an important risk factor for heart failure with little change over time. But why do the associations differ so substantially between the different measures of HbA1c? Single measurements of exposure are prone to substantial measurement errors and time-dependent fluctuations. Consequently, several epidemiological studies have shown that reliance on a single 'baseline' measure can substantially underestimate underlying associations when the aim is to estimate associations over longer exposure periods. ${ }^{4}$ In this context, it not very surprising to see that the updated mean $\mathrm{HbA1c}$ measure (which implicitly but not fully controls for regression dilution bias resulting from single measurements) shows a much stronger association than a single 
baseline $\mathrm{HbA1c}$ value. In fact, the strength of association from the repeated measure $\mathrm{HbA1c}$ associations is highly consistent with a meta-analysis of observational studies which showed an adjusted risk ratio of 1.15 (95\% confidence interval 1.10-1.21) for each 1 percentage point higher HbA1c. ${ }^{5}$ However, the totality of evidence to date without the present study has been based on about 14,000 heart failure events from 10 heterogeneous studies with no clear indication that these studies had controlled for regression dilution bias. ${ }^{5}$ Taken together, it seems appropriate to conclude that among patients with Type 2 diabetes each 1 percentage point higher 'usual' (i.e., long term average) HbA1c is associated with a 15\% higher risk of heart failure.

The next question raised by the study by Skrtic et al. is about the observed reverse association between latest $\mathrm{HbA} 1 \mathrm{c}$ measure and risk of heart failure at the lower end of the HbA1c spectrum. J-shaped associations are commonly encountered in medical literature and are often a reflection of reverse causality (that is the disease itself leading to low $\mathrm{HbA} 1 \mathrm{c}$ rather than the other way around). Previous studies have shown that when appropriate measures are taken to control for reverse causation (such as excluding the events in the first years after exposure), the J-shaped relationship often disappears. ${ }^{4}$ In this context, the authors are correct in urging us not to misinterpret the reverse association as evidence for harmful effects of aggressive glucose lowering. Whilst these findings suggest that in patients with established diabetes, a very low HbA1c could be seen as an indicator of higher risk, they do not permit any conclusions about treatment effects.

Indeed, the question of treatment effects and whether the associations between $\mathrm{HbA1c}$ and risk of heart failure are causal are still a matter of debate. Without doubt the positive association between hyperglycaemia and heart failure is at least in part due to residual uncontrolled confounding by common cardiovascular risk factors. However, there are several other plausible mechanisms that could be responsible for this association. These include processes such as insulin resistance or hyperinsulinaemia, endothelial dysfunction, 
dyslipidaemia, autonomic neuropathy, inflammation, hypercoagulability, and vascular calcification. Randomized trials, which by design are able to control for both known and unknown confounders, can sometimes help us to disentangle such complicated associations between risk factors and outcomes. However, for the question of hyperglycaemia and heart failure risk, we do now have any conclusive answers from randomized trials yet either.

Although proponents of the causal association between hyperglycaemia and risk of heart failure feel encouraged by the recent promising treatment effects of empagliflozin on risk of heart failure ${ }^{6}$ there are at least two reasons why the risk reductions observed in the trial are unlikely to be attributable to glycaemic control. The first reason is that empagliflozin's beneficial effects on heart failure were evident very early after initiation of therapy, suggesting that the mechanism of action for this effect might be related to the diuretic effect of the drug or its other short-term effects. The second reason is indirectly provided by the estimates of risk as measured by Skrtic et al. which indicate that the 35\% relative risk reduction in the trial is simply too strong to be attributable to its antiglycaemic effects. Even if we assume that the long-term association as measured by Skrtic et al. is causal, we would expect an intervention that is given over shorter term (in the EMPA-REG outcome trial 2.6 years) to reduce the risk of heart failure by no more than two-thirds of that the observed long-term association (i.e, about $7 \%$ to $10 \%$ relative reduction in risk of heart failure for each 1 percentage point reduction in $\mathrm{HbA1c}$ ). In the EMPA-REG outcome trial, empagliflozin reduced HbA1c by about 0.5 percentage point. ${ }^{6}$ Therefore, the reduction in risk of heart failure would be expected to be around or less than $10 \%$, which is substantially different from the actual $35 \%$ relative risk reduction reported in the trial.

Therefore, the conclusion from the Skrtic et al. to call for tighter control of blood glucose in patients with diabetes as a way of reducing the risk of heart failure seems premature. Until more convincing evidence becomes available, it seems prudent that we focus our efforts on 
133 better implementation of effective interventions for modifying risk, such as blood pressure 134 control $^{7}$ and cholesterol lowering treatment with statins ${ }^{8}$. 
137 1. Rubler S, Dlugash J, Yuceoglu YZ, Kumral T, Branwood AW, Grishman A. New type of cardiomyopathy associated with diabetic glomerulosclerosis. Am J Cardiol

2. Shah AD, Langenberg C, Rapsomaniki E, et al. Type 2 diabetes and incidence of 